\title{
Phenotypic and Genotypic Shifts in Hepatitis B Virus in Treatment-Naive Patients, Taiwan, 2008-2012
}

\author{
Chau-Ting Yeh, Kung-Hao Liang, \\ Ming-Ling Chang, Chao-Wei Hsu, Yi-Cheng Chen, \\ Chih-Lang Lin, Wey-Ran Lin, Ming-Wei Lai
}

We examined the characteristic changes of hepatitis $B$ virus (HBV) in antiviral drug treatment-naive patients referred for pretreatment evaluation in Taiwan during 20082012. Over time, we observed substantial decreases in the prevalence of $\mathrm{HBV}$ e antigen $(\mathrm{HBeAg})$ and increasing prevalence of the precore G1899A mutation and HBV-DNA levels in HBeAg-positive patients.

$\mathrm{H}$ epatitis B virus (HBV) replication is dependent on the activity of its reverse transcriptase, an errorprone enzyme, which results in the accumulation of genomic mutations. In the natural course of chronic hepatitis $\mathrm{B}$, the prevalence of some mutations (e.g., precore stop codon mutations, basal core promoter mutations, deletions in the pre-S gene region) gradually increases with the progression of disease through the different clinical stages $(1,2)$. This increase is largely caused by longer duration of chronic HBV infection, during which HBV has to adapt to environmental changes for better survival. Because of the availability of antiviral drug therapies, HBV replication can now be completely suppressed in most patients $(3,4)$. However, drug resistance and suboptimal or no responses to antiviral drugs can occur (5), and there is a lag of few months between the start of treatment and complete virologic suppression. In addition, treatment noncompliance and intermittent treatment almost always result in virologic, or even clinical, relapses. In such cases, antiviral drug therapy imposes an iatrogenic selection pressure on HBV, and the selected viruses carrying mutations can cause infections.

HBV has been infecting humans for a long time; thus, it is possible that genotypic or phenotypic changes have occurred over time, especially since the introduction of HBV vaccine and antiviral drugs. We examined serologic changes and genotypic alterations of HBV in treatment-naive patients during 2008-2012 in Taiwan, where a universal vaccination program was launched in 1986.

Author affiliation: Chang Gung Memorial Hospital, Taoyuan,

Taiwan

DOI: https://dx.doi.org/10.3201/eid2305.161894

\section{The Study}

During January 2008-December 2012, we reviewed the clinical and virologic data for 1,224 treatment-naive patients with chronic hepatitis B who were referred to our clinic at Chang Gung Memorial Hospital, Taoyuan, Taiwan, from all parts of Taiwan for pretreatment evaluation. We obtained patients' age and sex and assessed platelet count, cirrhosis status, HBV e antigen ( $\mathrm{HBeAg}$ ) and $\mathrm{HB}$ e antibody (antiHBe) status, HBV genotype, HBV DNA level, basal core promoter mutations, and precore stop codon mutations. We conducted the study using previously described methods $(6,7)$. The study was approved by the Institutional Review Board of Chang Gung Memorial Hospital.

Univariate regression analysis indicated a significant increase in patient age $(\mathrm{p}=0.001)$ and a significant decrease in the number of HBeAg-positive patients $(\mathrm{p}<0.001)$ over the 5-year year period. Three factors were significantly associated with $\mathrm{HBeAg}$ status: an increased prevalence of anti-HBe $(p=0.004)$ and increased prevalence of precore G1896A $(p=0.003)$ and G1899A $(p=0.019)$ mutations. However, no significant changes occurred in the prevalence of 9 of 10 basal core promoter mutations (online Technical Appendix Table 1, https://wwwnc.cdc.gov/EID/ article/23/5/16-1894-Techapp1.pdf). We noted a mild, but significant, decrease in the prevalence of G1730C mutations $(\mathrm{p}=0.034)$.

Multivariate analysis showed that patient age and changes in the prevalence of $\mathrm{HBeAg}$ were independent of each other (adjusted $\mathrm{p}=0.025$ and 0.021 , respectively). However, multivariate analysis that included G1730C, $\mathrm{HBeAg}$, and age showed that the changing prevalence of G1730C was not independent of $\mathrm{HBeAg}$ and age (adjusted $\mathrm{p}=0.222,0.049$, and 0.027 , respectively).

These data indicate that the decreasing prevalence of $\mathrm{HBeAg}$ was not due to the increasing age of treatmentnaive patients but due to an authentic phenotypic change of HBV over the years. It was unclear why patient age increased over the 5-year period; one possibility is the gradual acceptance of antiviral drug therapy by older patients, who may have been worried about possible side effects of new drugs. The cause of the decreasing prevalence of $\mathrm{HBeAg}$ over time was possibly due to the fact that a higher proportion of $\mathrm{HBeAg}$-positive patients were treated in the early era of antiviral drug use and more $\mathrm{HBeAg}$-negative patients received treatment at a later time. Alternatively, 
because HBV infection has occurred in and expanded among the human population over the past decades, the serologic alterations may be attributed to a changing trend in the mode of transmission on a population scale; for example, in Taiwan, horizontal transmission has increased because of injection drug abuse and sexual transmission, and vertical transmission has decreased because of the neonatal vaccination program.

We subsequently separated patients into HBeAg-positive $(n=398)$ and HBeAg-negative $(n=826)$ groups. In the $\mathrm{HBeAg}$-positive group, we saw a significant increase over time in the prevalence of precore G1899A mutations $(p=0.039)$ and level of HBV DNA $(p=0.013)$; however, these 2 factors were independent of each other (adjusted $p=0.009$ and 0.003 , respectively). Of note, we found no change in age over time in this subgroup $(\mathrm{p}=0.281)$ (online Technical Appendix Table 2). Furthermore, because these patients were all HBeAg-positive, we could not explain the data by a differential proportion of $\mathrm{HBeAg}$ positive patients being treated in the early and later periods of the antiviral drug era. Instead, other factors (e.g., changes in transmission routes, altered predominant risks of exposure, changes of HBV prevalence in different subpopulations) could be responsible. Alternatively, dependent on the scale of antiviral drug treatment received in this population, the therapeutic methods might also partly contribute to selection of mutation G1899A and HBV with higher replication efficiency.

In the $\mathrm{HBeAg}$-negative treatment-naive patients $(\mathrm{n}=$ $826)$, we found a borderline increase in patient age ( $\mathrm{p}=$ 0.046 ) and a borderline decreased prevalence of male patients $(p=0.044)$ over time. In addition, we noted a significant decrease in the prevalence of mutation A1752G over the 5 -year period $(\mathrm{p}=0.022)$. Multivariate analysis showed that these 3 changes were not independent (adjusted $p=$ 0.062 for age, 0.067 for male sex, and 0.201 for mutation A1752G; data not shown).

\section{Conclusions}

Our findings showed a shift in the phenotypic and genotypic characteristics of HBV in treatment-naive patients in Taiwan, an area where chronic hepatitis B is endemic, after the widespread use of antiviral drugs. In Taiwan, because of a limited budget for national health insurance and a high prevalence of chronic hepatitis $\mathrm{B}$, insurance coverage for hepatitis B treatment is not lifelong. Under the insurance plan, patients are provided with continuous nucleotide and nucleoside antiviral treatment for 3 years, after which the drugs are withdrawn to observe for durability of clinical remission. About half of patients have clinical relapses, and almost all have virologic relapses (8). Only patients with clinical relapses are retreated. After 3 years of retreatment, the antiviral drugs are with- drawn again to observe for durability; subsequent retreatments (for 3 years) are given only to patients with clinical relapses. The procedure is repeated until no clinical relapse has occurred. It is conceivable that the characteristics of HBV in these antiviral drug-treated, hepatitis Brelapsed patients have been selected by antiviral drugs. Antiviral drug-selected HBVs could potentially spread to treatment-naive patients and cause new infection or superinfection. However, the contribution of this therapeutic factor to the phenotypic and genotypic alterations of HBVs is unclear. Other environmental and social factors, such as altered transmission routes, changes of exposure risks, variations of HBV prevalence in subpopulations over time, and effects of neonatal vaccination, could lead to the changes in HBV characteristics.

Over the 5 years of this study, we found an increasing prevalence of G1899A mutations and an increasing concentration of serum HBV DNA in treatment-naive, HBeAg-positive patients. The mean HBV DNA concentrations increased from 7.7 to $8.3 \log _{10} / \mathrm{mL}$ and then stabilized without further increases during the last 3 years of the study. On the other hand, prevalence of G1899A mutations increased from $2.5 \%$ to $17.4 \%$. The clinical significance of this mutation in $\mathrm{HBeAg}$-positive patients is unclear and requires further study. In conclusion, this study revealed that, in Taiwan, HBV characteristics have been changing in the era after introduction of antiviral drug treatment and $\mathrm{HBV}$ vaccination.

\section{Acknowledgments}

We thank all staff of the Liver Research Center, Chang Gung Memorial Hospital, Taoyuan, Taiwan, for helpful discussions and technical assistance.

This study was supported by grants from Chang Gung Memorial Hospital Research Council (CRRPG3F0051 and CLRPG3C0012).

C.T.Y. designed and supervised the study; C.T.Y. and K.-H.L. drafted the manuscript; and M.-L.C., C.-W.H., Y.-C.C., C.-L.L., W.-R.L., and M.-W.L. collected the samples, performed all assays, and interpreted the data.

Dr. Yeh is director of the Liver Research Center and an attending physician in the Department of Hepato-gastroenterology, Chang Gung Memorial Hospital, Taoyuan, Taiwan. His primary research interests include molecular virology of hepatitis viruses and oncogenic mechanisms of hepatocellular carcinoma.

\section{References}

1. Yang HI, Yeh SH, Chen PJ, Iloeje UH, Jen CL, Su J, et al.; REVEAL-HBV Study Group. Associations between hepatitis B virus genotype and mutants and the risk of hepatocellular carcinoma. J Natl Cancer Inst. 2008;100:1134-43. http://dx.doi.org/ 10.1093/jnci/djn243 
2. Chen CH, Hung CH, Lee CM, Hu TH, Wang JH, Wang JC, et al. Pre-S deletion and complex mutations of hepatitis B virus related to advanced liver disease in $\mathrm{HBeAg}$-negative patients. Gastroenterology. 2007;133:1466-74. http://dx.doi.org/10.1053/j.gastro.2007.09.002

3. Ayoub WS, Keeffe EB. Review article: current antiviral therapy of chronic hepatitis B. Aliment Pharmacol Ther. 2011;34:1145-58. http://dx.doi.org/10.1111/j.1365-2036.2011.04869.x

4. Lok AS, McMahon BJ, Brown RS Jr, Wong JB, Ahmed AT, Farah W, et al. Antiviral therapy for chronic hepatitis B viral infection in adults: a systematic review and meta-analysis. Hepatology. 2016;63:284-306. http://dx.doi.org/10.1002/hep.28280

5. European Association for the Study of the Liver. EASL clinical practice guidelines: management of chronic hepatitis B virus infection. J Hepatol. 2012;57:167-85. http://dx.doi.org/10.1016/ j.jhep.2012.02.010

6. Yeh CT, So M, Ng J, Yang HW, Chang ML, Lai MW, et al. Hepatitis B virus-DNA level and basal core promoter A1762T/
G1764A mutation in liver tissue independently predict postoperative survival in hepatocellular carcinoma. Hepatology. 2010;52:1922-33. http://dx.doi.org/10.1002/hep.23898

7. Yeh CT, Chien RN, Chu CM, Liaw YF. Clearance of the original hepatitis B virus YMDD-motif mutants with emergence of distinct lamivudine-resistant mutants during prolonged lamivudine therapy. Hepatology. 2000;31:1318-26. http://dx.doi.org/10.1053/ jhep.2000.7296

8. Yeh CT, Hsu CW, Chen YC, Liaw YF. Withdrawal of lamivudine in $\mathrm{HBeAg}$-positive chronic hepatitis B patients after achieving effective maintained virological suppression. J Clin Virol. 2009;45:114-8. http://dx.doi.org/10.1016/j.jcv.2009.04.006

Address for correspondence: Chau-Ting Yeh, Liver Research Center, Chang Gung Memorial Hospital, 5 Fu-Shin St, Kuei-Shan District, Taoyuan, Taiwan; email: chautingy@gmail.com

\section{February 2015: Complicated Datasets}

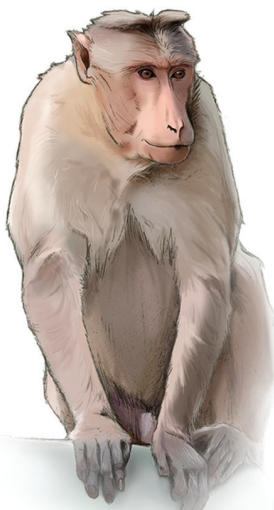

- Entry Screening for Infectious Diseases in Humans

- Timing of Influenza A(H5N1) in Poultry and Humans and Seasonal Influenza Activity Worldwide, 2004-2013

- Quantifying Reporting Timeliness to Improve Outbreak Control

- Tickborne Relapsing Fever, Bitterroot Valley, Montana, USA

- Simulation Study of the Effect of Influenza and Influenza Vaccination on Risk of Acquiring Guillain-Barré Syndrome

- Evidence for Elizabethkingia anophelis Transmission from Mother to Infant, Hong Kong
- Microbiota that Affect Risk for Shigellosis in Children in Low-Income Countries

- Optimizing Distribution of Pandemic Influenza Antiviral Drugs

- pH Level as a Marker for Predicting Death among Patients with Vibrio vulnificus Infection, South Korea, 2000-2011

- Refining Historical Limits Method to Improve Disease Cluster Detection, New York City, New York, USA

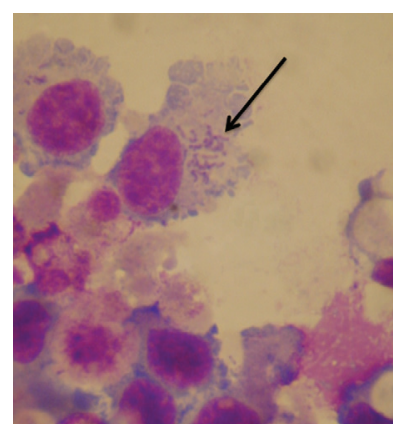

- Naturally Acquired Antibodies against Haemophilus influenzae Type a in Aboriginal Adults, Canada

- Infectious Causes of Encephalitis and Meningoencephalitis in Thailand, 2003-2005

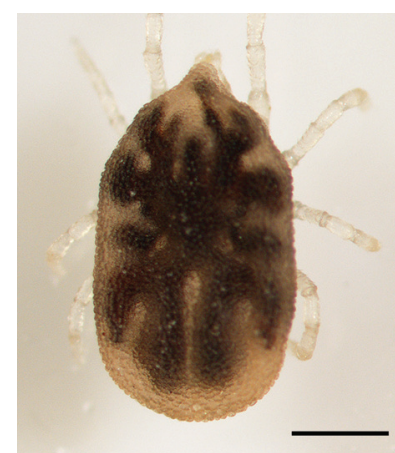

- Lagenidium giganteum Pathogenicity in Mammals

- Novel Reassortant Influenza $\mathrm{A}(\mathrm{H} 5 \mathrm{~N} 8)$ Viruses among Inoculated Domestic and Wild Ducks, South Korea, 2014

- Vesicular Stomatitis VirusBased Vaccines against Lassa and Ebola Viruses

- Use of Insecticide-Treated House Screens to Reduce Infestations of Dengue Virus Vectors, Mexico

- Comparative Analysis of African Swine Fever Virus Genotypes and Serogroups

- Murine Typhus, Reunion, France, 2011-2013

- Awareness and Support of Release of Genetically Modified "Sterile" Mosquitoes, Key West, Florida, USA
- Novel Candidatus Rickettsia Species Detected in Nostril Tick from Human, Gabon, 2014

- Outbreak of Henipavirus Infection, Philippines, 2014

- Ascariasis in Humans and Pigs on Small-Scale Farms, Maine, USA, 2010-2013

- Potentially Novel Ehrlichia Species in Horses, Nicaragua

- Neisseria meningitidis ST-11 Clonal Complex, Chile 2012

- Molecular Diagnosis of Cause of Anisakiasis in Humans, South Korea

- Streptococcus suis Infection in Hospitalized Patients, Nakhon Phanom Province, Thailand

- Exposure-Based Screening for Nipah Virus Encephalitis, Bangladesh

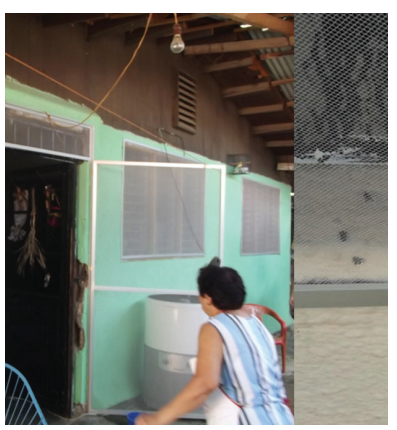

